\title{
La tutoría académica en la educación superior. Una investigación a partir de entrevistas y grupos de discusión en la Universidad de Cantabria (España)
}

\section{Academic mentoring in higher education. A research based on interviews and discussion groups at the University of Cantabria (Spain)}

Carlos RODRÍGUEZ-HOYOS, Adelina CALVO SALVADOR e Ignacio HAYA SALMÓN Universidad de Cantabria

Recibido: Diciembre 2013

Aceptado: Febrero 2014

\section{Resumen}

En este artículo presentamos los principales resultados de una investigación en proceso dirigida a comprender la visión que los estudiantes de los primeros cursos de Grado tienen sobre la tutoría en la universidad. De forma más concreta, este trabajo se centra en el análisis de la tutoría académica. Para ello se ha utilizado un diseño cualitativo de investigación y se ha seleccionado una muestra de estudiantes cuyas titulaciones abarcan las cinco áreas de conocimiento en las que se organizan sus estudios. Los resultados obtenidos permiten identificar algunos problemas en el desarrollo de la tutoría académica, como las debilidades que presenta la orientación académica en la transición de los jóvenes a la universidad, la necesidad de integrarla en el espacio del aula o su utilización para mejorar la evaluación de la docencia. La tutoría académica se presenta como un instrumento que podría facilitar la mejora e innovación de los procesos de educación superior. El trabajo se ha desarrollado en la Universidad de Cantabria durante los cursos académicos 2011-12 y 2012-13.

Palabras clave: tutoría académica en la universidad, mejora de la docencia, entrevistas, grupos de discusión.

\begin{abstract}
This paper shows the main results of an ongoing research aimed to understand the view that students have about mentoring at university, in their first year degree. More specifically, this work focuses on the analysis of academic mentoring. To do this we used a qualitative research design and selected a sample of students from the five areas of knowledge offered in our university. The results allow us to identify some problems in the development of mentoring and the weaknesses related to the academic orientation in the transition from secondary school to university, the need to integrate it into the classroom space or its potential use in order to improve the assessment of teaching and learning. Academic mentoring is presented as a tool that could promote the improvement and innovation of higher education processes. The work
\end{abstract}


has been developed at the University of Cantabria during the academic years 2011-12 and 201213.

Keywords: academic mentoring at university, teaching improvement, interviews, focus groups.

La implantación del Espacio Europeo de Educación Superior (EEES) en nuestro país ha supuesto la emergencia de diferentes debates académicos en torno a temas diversos que afectan a la estructura, el currículum o la cultura de las instituciones de educación superior. Entre todos ellos se encuentran los que están dirigidos a discutir y valorar las dimensiones conceptuales y organizativas de la orientación universitaria y, más concretamente, de la acción tutorial (Álvarez, 2008; Gairín, et al., 2004; García Nieto et al., 2005; García Nieto, 2008). La comunidad docente e investigadora ha expuesto la complejidad de este concepto en trabajos que han profundizado, tanto en las diferentes formas en la que ésta se concibe desde una perspectiva teórica, como en las maneras de concretar sus dimensiones en los centros de educación superior (Sobrado, 2008).

Nuestro trabajo se centra en la dimensión académica de la tutoría en la universidad y persigue el propósito de adquirir un conocimiento profundo de las experiencias que los jóvenes estudiantes están teniendo en la institución universitaria. Dado que la tutoría es inherente a la función docente, entendemos que este conocimiento nos permite hacer una primera valoración sobre algunos aspectos que se han considerado centrales en la reciente Reforma Universitaria como el trabajo por competencias, la evaluación de la docencia o las nuevas metodologías docentes más activas (Castaño et al., 2007; Giné, 2009; Vallejo y Molina, 2011).

Si bien existen diferencias de matiz entre las definiciones aportadas por los diversos autores, existe un cierto consenso a la hora de señalar que la acción tutorial se desarrolla a través de tres dimensiones (personal, académica y socioprofesional) y que entre sus principales finalidades está la de facilitar el desarrollo integral de los estudiantes, favorecer la personalización de los procesos educativos, mejorar los entornos de aprendizaje, dinamizar las relaciones de aula o promover la participación de los jóvenes en las instituciones (Álvarez y Jiménez, 2003; Rodríguez, 2004).

A pesar de que la tutoría pueda adoptar en la universidad diferentes formas, su objetivo se dirige a la promoción del desarrollo integral del alumnado y es considerada un componente intrínseco de la enseñanza (Herrera, 2011). Por otro lado, hoy parece indudable que su desarrollo tiene efectos positivos para los estudiantes, el profesorado y la institución. Así, para el alumnado supone una fuente importante de información, asesoramiento y orientación a lo largo de su proceso formativo, permitiéndole desarrollar las competencias indispensables para el desarrollo de una ciudadanía plena (trabajo en equipo, colaboración, etc.). Desde el punto de vista del profesorado, la tutoría debe suponer una oportunidad para mejorar los procesos de colaboración docente, para aumentar el grado de información sobre su titulación, sobre el plan o planes de estudio en los que participa, etc. Se considera, también, que es una oportunidad para establecer cauces de evaluación y reflexión sobre su docencia, así como una vía para democratizar los procesos educativos, dando un papel más activo a 
los estudiantes. Por último, la tutoría en la universidad tiene efectos positivos para la propia institución dado que permite detectar necesidades que permanecen ocultas, así como abrir diferentes canales de comunicación entre los distintos miembros de la comunidad universitaria, tanto hacia el interior de la misma, como hacia el exterior (Calvo, Haya y Rodríguez-Hoyos, 2012).

Los procesos de orientación y tutoría se conciben como elementos centrales en el Espacio Europeo de Educación Superior, considerando su desarrollo indispensable para alcanzar el cambio filosófico y metodológico que la reforma europea propone. Se entiende, así, que la tutoría forma parte de la función docente y que debe estar dirigida a favorecer el trabajo individual y grupal de los estudiantes. Con el objetivo de que los jóvenes universitarios sean cada vez más autónomos en los procesos educativos, la tutoría ayuda al desarrollo de metodologías docentes más activas como el aprendizaje por descubrimiento o a través de procesos de investigación. Supone, en definitiva, reflexionar no sólo sobre la enseñanza sino también, y sobre todo, sobre el aprendizaje, lo que sin duda nos lleva a la creación de un nuevo profesional docente en la educación superior (Martínez Serrano, 2009; Rumbo y Gómez, 2011; Sola y Moreno, 2005).

Hasta este momento, la dimensión académica de la acción tutorial es la que ha tenido una mayor presencia en las instituciones de educación superior y, generalmente, suele desarrollarse a través de actividades individualizadas en las que el profesorado trabaja con el alumnado para mejorar los procesos de enseñanza-aprendizaje (Arbizu, Lobato y Castillo, 2005; Dopico, 2013). Si bien algunos autores han puesto de manifiesto la necesidad de concebir la tutoría académica como un aspecto más de la docencia (Álvarez y González, 2008), algunos trabajos siguen mostrando que esa integración no llega a producirse y que el alumnado percibe la acción tutorial como una ayuda complementaria a las asignaturas (García y Troyano, 2009).

Dado que para tener un conocimiento profundo sobre cómo se desarrolla la tutoría en la educación superior necesitamos conocer el punto de vista de todos sus protagonistas, una de las líneas de investigación más fructíferas en este campo es la que se dirige a analizar las percepciones de los estudiantes sobre los planes de acción tutorial. Un ejemplo de estos trabajos es el de Rumbo y Gómez (2011) que muestra el elevado grado de satisfacción del alumnado que participó en el plan de acción tutorial de la Universidad de A Coruña o el de Álvarez, Lorca y García (2010), que pone de manifiesto la baja participación del colectivo discente en las actividades de acción tutorial y su baja satisfacción con la información académica recibida.

Por otro lado, existen un conjunto de trabajos que han analizado cómo valora el alumnado la docencia en el marco del EEES, identificándose cuestiones clave como la visión que tiene este colectivo de las competencias que se trabajan en la universidad y su importancia en el entorno laboral (De La Iglesia, 2012) o la importancia que concede a las metodologías docentes activas en el aula (Ruiz De Miguel y Oliveros, 2006; Vallejo y Molina, 2011).

Otra de las líneas de investigación más desarrolladas en nuestro país es la que se dirige a analizar la concepción que el profesorado tiene sobre la tutoría académica en la educación superior. El trabajo de Álvarez y González (2005) reflejó que si bien este 
colectivo entendía la tutoría como una actividad más dentro los procesos de enseñanzaaprendizaje, en realidad no se procede a su integración dentro del desarrollo de las asignaturas en las que participa, por lo que, en definitiva, tiene dificultades para contribuir a la mejora de los aprendizajes del alumnado.

Con todo ello, a través de una investigación cualitativa, hemos abierto un diálogo con los estudiantes para conocer sus experiencias y opiniones sobre la tutoría, la docencia y la universidad. De esta forma, hemos considerado a los estudiantes como “informantes expertos" en este área, situándonos en la línea de otros trabajos que, tanto en el panorama nacional (Giné, 2009; Hernández et al., 2011) como en el internacional (Hayes y Ljungberg, 2011; Seale, 2010) tratan de indagar en determinados aspectos de los procesos de educación superior desde la experiencia y visión de los aprendices, otorgándoles un rol mucho más activo.

\section{Diseño de la investigación, metodología y análisis de datos}

Nuestra investigación se enmarca en el paradigma cualitativo de investigación (Bisquerra, 2004; Sandín, 2003). El hecho de que hayamos optado por este enfoque se debe a que dentro de nuestros intereses se encontraba llegar a entender cómo interpretan los estudiantes las actividades de acción tutorial en las que participan. Consideramos que investigar sobre la dimensión académica de la acción tutorial exige sumergirse en las visiones que el alumnado construye sobre la docencia, dado que cada una de estas visiones aporta información novedosa, y en parte original, sobre la concepción de los procesos de enseñanza-aprendizaje en el marco del EEES. Este paradigma nos permite acercarnos al objeto de estudio explorando la reconstrucción subjetiva que el alumnado realizaba sobre las prácticas docentes y reconociendo la diversidad de los ambientes sociales en los que se desarrollan esas actividades (Flick, 2009).

En ese sentido, nuestro trabajo se complementa con aquellos de corte más cuantitativo destinados a realizar una descripción general de las percepciones del alumnado sobre la tutoría académica, dado que pretende dar un paso más y complementar esas propuestas, llegando a comprender cómo se desarrolla la dimensión académica de la tutoría en las aulas. Eso nos ha llevado a focalizar esta investigación en aquellas interpretaciones y significados que los participantes dan a sus propias experiencias en contextos educativos diversos y singulares. Entendemos, pues, que la comprensión sobre un fenómeno sumamente complejo que tiene tonalidades diferentes en cada espacio educativo ha de combinarse con las aportaciones de aquellos trabajos que tratan de ofrecer una visión macroestructural sobre el objeto de estudio, para imaginar acciones encaminadas a mejorar la acción tutorial en la educación superior (García, 2011).

La técnica utilizada para la selección de la muestra fue la bola de nieve. La muestra utilizada en la investigación se compuso de un total de 13 estudiantes (8 mujeres y 5 hombres). En el momento de la recogida de datos, todos ellos se encontraban cursando el primer o segundo curso de alguna de las titulaciones impartidas en la Universidad de Cantabria. La selección de las titulaciones se hizo en relación a la accesibilidad de la 
muestra y con el objetivo de lograr que todos los campos de conocimiento en los que se organizan las titulaciones de nuestra universidad (cinco) estuvieran representados.

De esta forma, en la investigación participaron estudiantes de cinco titulaciones que representan a otros tantos campos de conocimiento: Historia (por la rama de Artes y Humanidades), Matemáticas (rama de Ciencias), Enfermería (Ciencias de la Salud), Maestro Educación Primaria (Ciencias Sociales y Jurídicas) y Recursos Energéticos (Ingeniería y Arquitectura).

En relación a las técnicas de recogida de datos, hemos utilizado grupos de discusión y entrevistas (Barbour, 2013; Kvale, 2011), considerando que las mismas son esenciales cuando intentamos penetrar en los significados que los protagonistas atribuyen a las actividades y dinámicas sociales en las que han participado y de las que forman parte. Nos permite, también, ahondar en las concepciones y vivencias particulares que éstos han tenido en su paso por la universidad. En este sentido, hemos considerado los textos generados a partir de las entrevistas y grupos de discusión como narraciones a partir de las cuales comprender cómo se desarrolla la acción tutorial en la educación superior, con un grupo de sujetos concretos y en un lugar y tiempo particular. Estas narraciones surgen en la interacción entre investigadores y participantes y suponen una forma de pensamiento y la expresión de una determinada forma de mirar el mundo.

Todas las entrevistas y grupos de discusión se realizaron a partir de un guión elaborado previamente, por lo que las técnicas tuvieron un carácter semi-estructurado. Las preguntas formuladas fueron agrupadas en diferentes bloques temáticos: datos identificativos de los participantes; la transición entre la educación secundaria y superior; los procesos de enseñanza-aprendizaje y la tutoría en la universidad y, finalmente, la participación y su relación con la tutoría.

El análisis de datos se realizó a través de un proceso de codificación temática (Angrosino, 2012; Flick, 2009) aplicado a las transcripciones obtenidas tras la aplicación de las técnicas de recogida de datos.

A continuación presentamos un resumen de la muestra, técnicas de recogida de datos y titulaciones y ramas de conocimiento (tabla 1):

\begin{tabular}{|c|l|c|}
\hline Muestra & \multicolumn{1}{|c|}{ Titulación/Área de conocimiento } & Técnica empleada \\
\hline 2 & Estudiantes de Grado en Historia/ Artes y Humanidades & Entrevista \\
\hline 3 & Estudiantes de Grado en Matemáticas/Ciencias & Grupo de discusión \\
\hline 1 & Estudiante de Grado en Enfermería/ Ciencias de la Salud & Entrevista \\
\hline 5 & $\begin{array}{l}\text { Estudiantes de Grado en Magisterio/ Ciencias Sociales y } \\
\text { Jurídicas }\end{array}$ & Grupo de discusión \\
\hline 2 & $\begin{array}{l}\text { Estudiantes de Grado en Recursos Energéticos/ Ingeniería y } \\
\text { Arquitectura }\end{array}$ & Entrevista \\
\hline
\end{tabular}

Tabla 1. Perfil de los participantes y técnicas empleadas

\section{Resultados}

El análisis de los datos recogidos nos ha permitido identificar tres grandes ideas a partir de las cuales discutir la dimensión académica de la tutoría en la universidad: la transición desde la educación secundaria a la educación superior, la tutoría académica 
en el aula y la evaluación de la docencia por parte del alumnado como estrategia de mejora de la enseñanza.

\section{La débil orientación académica en la transición a la universidad}

En este epígrafe analizaremos qué tipo de orientación académica ha recibido el alumnado en el momento previo a la elección de la titulación que va a cursar. Si bien esta orientación se desarrolla en los centros de secundaria, consideramos que es esencial profundizar en sus principales características para mejorar el tipo de estrategias que se ponen en marcha desde la universidad dado que, como señalaremos, ésta se acerca a los institutos para trasladar su oferta académica a los futuros/as universitarios/as. Los datos extraídos nos sugieren que sería necesario repensar el tipo de relación que se establece entre estos dos niveles educativos en aras a favorecer la transición académica.

Los estudiantes reconocieron, de forma mayoritaria, que su primer contacto con el mundo universitario fue, bien a través de visitas a las Facultades, bien a través de diferentes actividades puntuales como charlas organizadas por el departamento de orientación de su centro. Entre todas estas estrategias destaca la labor de las universidades privadas en el proceso de captación de alumnado:

"A nosotros, más que nada se nos acercaban universidades privadas, de Madrid, de Barcelona y, sin embargo, de la universidad de Cantabria tampoco nos dieron mucha información (...) [Nos dieron información], sí, de la selectividad y de cómo había que hacerla, eso sí” (GD Recursos energéticos).

Sin lugar a dudas, este tipo de afirmaciones nos llevan a preguntarnos, por un lado, qué visibilidad están teniendo las universidades públicas en los centros de secundaria y, por el otro, qué tipo de información académica se ofrece al alumnado para que pueda realizar una elección informada. Resulta significativo que algunas de las intervenciones de los participantes dejaran entrever que la información ofrecida por las universidades sobre sus planes de estudio en las charlas o jornadas de puertas abiertas podría resultar insuficiente para que éste realizara una elección informada. En este sentido, señalan:

"[Desde la universidad] Venían a explicarte qué era la PAU o había actividades en plan... pero vamos, como lo de las visitas a la universidad, pero nada que me hiciese decantarme por Matemáticas” (GD Matemáticas).

Al mismo tiempo, los participantes en esta investigación han coincidido en señalar que la estrategia más común de orientación académica desarrollada en los centros de secundaria es la aplicación e interpretación de pruebas psicométricas, paso previo para informar a los estudiantes sobre las opciones académicas que mejor se adaptan a sus capacidades e intereses:

"Además en $4^{\circ}$ de la ESO, por lo menos en mi instituto, te hacían como un test para ver por qué rama te debías de orientar en Bachiller, ver qué especialidad se te daba mejor y eso. (...) Sí, nosotros hicimos uno [cuestionario] que era sobre lo que supuestamente más te gustaba” (GD Historia). 
Tal y como se deriva de sus intervenciones, no parece que la realización de esas pruebas se acompañara, en la mayor parte de los casos, del desarrollo de otro tipo de estrategias que permitiera a los estudiantes realizar una elección más informada, como, por ejemplo, las materias y contenidos que se imparten en las diferentes titulaciones, las posibles salidas profesionales, etc. En este sentido, la orientación académica parece tener lugar en la educación secundaria de forma paralela al currículum a través de acciones puntuales en los considerados "momentos críticos" de la trayectoria académica de los jóvenes.

En definitiva, hemos podido constatar cómo las relaciones que se establecen entre los centros de secundaria y la institución universitaria para acompañar la transición académica entre estas dos etapas no parecen estar facilitando una toma de decisiones informada. Al mismo tiempo, es necesario realizar un mayor esfuerzo para visibilizar la oferta de educación pública en la etapa universitaria.

\section{La dimensión académica de la tutoría en el espacio del aula}

Uno de los aspectos en los que ha coincidido mayoritariamente el alumnado entrevistado ha sido en señalar la utilización en las clases de estrategias metodológicas que este colectivo interpreta como incompatibles o inadecuadas con la implantación del EEES. Si bien reconocen la existencia de algunos cambios en la definición de las estrategias de evaluación, en los que se otorga una mayor relevancia a los trabajos y prácticas de aula, el tipo de estrategias metodológicas siguen siendo de naturaleza eminentemente transmisiva, en las que sigue dominando la utilización de exposiciones magistrales por parte del profesorado. Con ello, los estudiantes consideran que se mantiene la misma forma de dar clase que se daba en las antiguas licenciaturas, donde la mayor parte del tiempo asisten a una clase magistral (GD Historia).

En segundo lugar, la mayoría de los participantes coinciden en señalar que si bien en algunas de las asignaturas se combina el trabajo individual y el trabajo cooperativo, sigue predominando el primero frente al segundo. Al mismo tiempo, los jóvenes señalan las dificultades con las que se encuentran a la hora de desarrollar actividades grupales, debido a la escasa implicación de algunos miembros del grupo, la falta de tiempo para reunirse fuera del horario de las asignaturas o a la ausencia de estrategias del profesorado para enseñar cómo se trabaja de forma colaborativa. En ese sentido, reconocen que una de las prácticas más frecuentes es la distribución de los trabajos en partes entre todos los miembros del grupo que, finalmente, ensamblan en un producto común. Los jóvenes afirman que el profesorado no desarrolla en el aula estrategias que permitan mejorar sus competencias para el trabajo en equipos (GD Magisterio, GD Matemáticas). De hecho, esa escasa preparación para el trabajo grupal supone una desatención a las competencias, ya que saber trabajar en equipo es una de las competencias más demandadas en el actual mercado laboral.

En tercer lugar, los datos recogidos en las distintas entrevistas y grupos de discusión ponen de manifiesto las dificultades con las que, generalmente, se encuentra el alumnado a la hora de cursar una asignatura en la que interviene más de un docente. Los estudiantes evidenciaron que la falta de coordinación dificulta enormemente el 
desarrollo de estrategias de orientación académica dentro de las asignaturas e, incluso, en el propio plan de estudios:

"A mí me gustaría que algunas cosas estuviesen más organizadas y fuesen más coherentes como, por ejemplo, los horarios, las asignaturas... que igual están un poco desorganizadas, entre ellos mismos [los profesores] no saben muy bien qué le toca a cada uno dar cuando hay varios profesores” (GD Matemáticas).

Finalmente, el alumnado reconoce la importancia del desarrollo de una adecuada labor de tutorización académica en el seno de cada una de las asignaturas que cursan para poder superarlas con éxito. Así, revelan la importancia que para ellos adquiere asistir a sesiones en las que el profesorado asesore y acompañe el desarrollo de las diferentes actividades de un modo cercano y continuo. De esta forma, a propósito de la elaboración de un trabajo grupal señalan:

“...En ese caso sí estábamos asesorados porque nos obligaban a ir a una tutoría, pero el resto de casos [en los que teníamos que hacer] trabajos [en grupo], de asesoramiento tuvimos poquito. Nos dicen "tenéis que hacer esto, y ya” (GD Magisterio).

Con ello, hemos constatado que el alumnado identifica el apoyo o asesoramiento académico como un factor clave en su formación que debería integrarse en las estrategias metodológicas de aula.

Por otro lado, los datos recogidos también permiten afirmar que el alumnado se siente más cómodo con estilos docentes en los que se posibilita el establecimiento de relaciones pedagógicas fluidas, cercanas y continuas que favorezcan la integración de la dimensión académica de la tutoría en el desarrollo de las diferentes asignaturas:

"Los profesores eran muy cercanos todos. Hemos tenido mil tutorías para preguntar lo que quisiésemos. Yo, desde luego, de los profesores no tengo ninguna queja. Todo lo contrario, nos han ayudado todo lo que han podido. Y nos han puesto clases de refuerzo si nos hacían falta en verano, sus correos electrónicos siempre estaban disponibles” (GD Recursos energéticos).

En resumen, las intervenciones del alumnado señalan algunos aspectos a mejorar en el ámbito de la tutoría académica, como serían la toma de decisiones sobre las estrategias metodológicas, el desarrollo de las competencias (como la de trabajar en equipo) o la coordinación docente. Al mismo tiempo, los jóvenes reconocen la importancia de mantener estilos docentes en los que se integre la dimensión académica de la tutoría dentro del espacio cotidiano del aula.

\section{La evaluación de la docencia por parte del alumnado como estrategia de mejora de la enseñanza}

En nuestra investigación, el colectivo de estudiantes se ha mostrado crítico con el tipo de instrumentos que se están utilizando para recoger sus opiniones y valorar la calidad de la docencia y tutoría que reciben, aunque no dudan en ningún momento de la evaluación como instrumento para producir mejoras. 
Así pues, podemos afirmar que la evaluación de la docencia por parte de los estudiantes es, sin lugar a dudas, una cuestión que éstos entienden como necesaria para mejorar los procesos de diseño y desarrollo curricular de las diferentes asignaturas:

"Me parece muy bien porque hay que contar con la opinión de los alumnos, no siempre es lo que el profesor diga, sino que ellos también pueden corregir, errores tenemos todos, y como ellos dicen, a veces ellos los primeros, y entonces también les ayuda... pues a mejorar en su enseñanza” (Entrevista Enfermería).

Conviene puntualizar que si bien el colectivo discente reconoce la relevancia de la evaluación de la docencia, que en la Universidad de Cantabria se realiza a través del Sistema de Garantía Interno de Calidad, no conoce cuáles son los resultados de sus opiniones ni la finalidad para la que se utilizan esos datos. En este sentido, los estudiantes son bastante pesimistas con respecto al verdadero impacto que sus opiniones tienen a la hora de introducir mejoras en las diferentes dimensiones de las asignaturas:

“Eso, por ejemplo, [que el profesor llega todos los días 30 minutos tarde] todo el mundo lo habrá escrito en el cuestionario de evaluación, pero no se sabe si el año que viene va a valer de algo haberlo puesto o no. No está claro” (GD Recursos energéticos).

Por otro lado, manifestaron que los cuestionarios diseñados resultan demasiado cerrados y no permiten reflejar la complejidad de sus verdaderas opiniones sobre los aspectos evaluados. Reconocieron, además, las dificultades con las que se encuentran para interpretar algunos ítems, lo que les lleva a recurrir, en ocasiones, a estrategias como responder a las preguntas al azar sin fijarse en su contenido. Paralelamente, propusieron sustituir la técnica del cuestionario por otras en las que pudieran expresar con mayor libertad sus opiniones sobre las dimensiones evaluadas:

"Lo mejor [del cuestionario] es el apartado de las observaciones [un apartado final abierto] cuando un profesor de verdad tiene algo que quieres criticar o alabar, lo que importan son las observaciones porque las preguntas en sí pues tampoco nos sacan de mucho. Hay veces que la gente los rellena al azar [los ítems cerrados]" (GD Matemáticas).

Pese a todo, el alumnado es capaz de imaginar algunas propuestas de mejora que se orientan a dar valor a la evaluación formativa de la docencia, como la que se dirige a abrir un espacio de diálogo entre el profesorado y sus estudiantes, en el caso de que éste obtuviera "bajas calificaciones" en su evaluación (GD Magisterio). Estas intervenciones revelan, sin duda, que el alumnado concibe la evaluación como un espacio destinado a la mejora de la práctica docente en sus múltiples dimensiones.

\section{Discusión de resultados y conclusiones}

En este artículo hemos presentado los resultados de una investigación centrada en conocer la opinión de los jóvenes universitarios sobre la tutoría académica en la universidad. Así, hemos podido conocer los relatos y valoraciones que éstos realizan 
sobre las actividades de orientación en las que participaron durante su transición a la universidad. Hemos constatado que en la toma de decisiones durante la elección de los estudios superiores no jugó un papel especialmente importante el proceso de orientación y tutoría que tuvo lugar durante el final de su educación secundaria, saliendo a la luz que ésta se hizo visible sólo mediante actividades puntuales (charlas, jornadas de puertas abiertas, tests, etc.) en los momentos que consideramos "críticos" por ser los tiempos en los que los estudiantes tienen que decidir entre distintas opciones académicas y/o socioprofesionales.

Junto con ello, se constata todavía una presencia débil de las instituciones públicas de educación superior en su acercamiento a la educación secundaria, siendo esta mucho más fuerte en el caso de las instituciones privadas, más acostumbradas a la política del marketing y de "captación de clientes".

En consonancia con los resultados de otras investigaciones (Domínguez, Álvarez y López, 2013), este trabajo ha puesto de manifiesto que resulta primordial estrechar los lazos entre ambos niveles educativos para mejorar la información y orientación académica del alumnado, ya que, tal y como hemos visto, en demasiadas ocasiones los jóvenes apenas conocen qué van a encontrarse cuando den el salto a la educación superior. Y esto debería suponer una mejora en el sistema de orientación y tutoría en los dos niveles educativos. En la secundaria, éste deberían estar más vinculado con el currículum (Fernández-Sierra, 1993), y en la universidad, éste debería comenzar a entenderse como un elemento que favorece los aprendizajes de los estudiantes y posibilita la coordinación docente (Herrera, 2011).

En segundo lugar, esta investigación nos ha permitido demostrar que el alumnado se convierte en un interlocutor imprescindible para lograr cambios en la acción tutorial en el contexto universitario (Calvo, Haya y Rodríguez-Hoyos, 2012; Giné, 2009). El diálogo abierto con este colectivo ha permitido identificar algunos aspectos a mejorar dentro de la actividad docente desarrollada en las titulaciones de las diferentes áreas de conocimiento de la Universidad de Cantabria como, por ejemplo, la transformación de las estrategias metodológicas (hacia un mayor protagonismo de los estudiantes) o la necesidad de seguir haciendo hincapié en la importancia de trabajar las competencias (algo fundamental, no sólo para el acceso al mercado laboral, sino en la asunción del rol de ciudadanos activos y críticos). Los resultados obtenidos nos sugieren que es necesario repensar la concepción de la acción tutorial como estrategia de acompañamiento del alumnado en los procesos de enseñanza-aprendizaje, concibiéndola como un elemento que no debería desarrollarse únicamente a través de actividades paralelas a la docencia y entendiéndose como algo inherente a la función docente (Álvarez, 2008; Crisol, 2012; Dopico, 2013; Lobato y Livento, 2013; Pérez Cuso, 2013).

Se entiende, así, que la tutoría académica ha de concebirse como una oportunidad para establecer un diálogo con el alumnado que nos permita, por un lado, potenciar los procesos de coordinación docente y, por el otro, imaginar procesos de innovación educativa, entendidos como cambios planeados a pequeña escala, adaptados al contexto en el que se desarrollan y que supongan una mejora en las prácticas educativas (Rodríguez Romero, 2003). Estos procesos permitirían desarrollar un 
conocimiento más profundo sobre nuestra propia práctica docente, elaborar una perspectiva más compleja sobre la realidad institucional o facilitar la reflexión y el análisis sistemático de las vivencias que tienen lugar en las aulas.

Si bien reconocemos la importancia de las iniciativas institucionales destinadas al diseño y desarrollo de planes de acción tutorial concebidos desde los órganos de gestión universitarios (Cano, 2008), es necesario forjar otros modelos que sigan una lógica de abajo-arriba. Eso supone otorgar un mayor protagonismo al profesorado a la hora no sólo de gestionar la acción tutorial que ya ha sido definida desde instancias institucionales, sino también de diseñar las actividades encaminadas a trabajar las diferentes dimensiones de la tutorización del alumnado que más se ajusten a las necesidades de su contexto. No podemos obviar que la práctica educativa es inherentemente inestable y que la única forma de dar respuesta a esa incertidumbre es desarrollar un modelo de profesional docente con capacidad de indagación que pueda ir incrementando y mejorando los conocimientos sobre su trabajo en un entorno que favorezca y permita la colegialidad. Para ello, es necesario que las propias universidades adopten algunas medidas encaminadas a mejorar la formación de sus docentes en esta materia, así como a desarrollar políticas de reconocimiento para los docentes que ya vienen desarrollando estas labores (Sobrado, 2008; Martínez Muñoz, 2009).

Finalmente, este trabajo ha revelado la necesidad de reflexionar sobre las estrategias que las universidades están empleando para evaluar la docencia universitaria, dado que podrían estar convirtiéndose en procesos burocratizados que no permitirían responder a su fin último, que no es otro que el de la mejora de la enseñanza. Consideramos que la dimensión académica de la tutoría podría convertirse en una estrategia privilegiada para hacer posible una verdadera evaluación para la mejora (Escudero, 2000). En este sentido, la evaluación de la práctica docente ha de ser una de las metas de la tutoría académica, por lo que es necesario idear acciones que posibiliten la recogida de datos sobre el desarrollo de los procesos de enseñanza-aprendizaje para adoptar medidas encaminadas a mejorarlos. Por otro lado, la dimensión académica se convierte en un espacio privilegiado para indagar sobre la pertinencia de las estrategias empleadas para evaluar (metaevaluación), tanto los aprendizajes del alumnado, como los procesos educativos.

\section{Limitaciones del estudio y prospectiva}

A la luz de los resultados obtenidos en nuestro estudio parece conveniente seguir investigando sobre la situación real de la dimensión académica de la tutoría en la universidad y sobre sus potencialidades para mejorar los procesos educativos y la situación del alumnado en la institución, teniendo como horizonte construir instituciones más participativas. En este sentido, sería necesario ampliar la muestra a otras titulaciones y seguir orientando la investigación hacia la búsqueda de mejoras en las actividades y planes de acción tutorial.

Por otro lado, es importante seguir desarrollando investigaciones en este ámbito con una metodología cualitativa o mixta, de manera que logremos tener una visión más 
compleja y completa de la tutoría en la universidad en cualquiera de sus ámbitos (personal, académico y socioprofesional). Sin duda, las investigaciones precedentes y la que hemos presentado aquí podrían inspirar a las diferentes universidades españolas en el proceso de acometer la tarea de valorar sus actividades, planes y programas de tutoría en la educación superior.

\section{Referencias bibliográficas}

ÁLVAREZ, M. (2008). La tutoría académica en el Espacio Europeo de la Educación Superior. Revista Interuniversitaria de Formación del Profesorado, 22 (1), 71-88.

ÁLVAREZ, P. Y GONZÁLEZ, M. (2005). La tutoría académica en la enseñanza superior: una estrategia docente ante el nuevo reto de la convergencia europea. Revista Interuniversitaria de Formación del Profesorado, 8 (4), 1-4.

ÁLVAREZ, P. Y JIMÉNEZ, H. (2003). La intervención tutorial en la enseñanza universitaria, en P. Álvarez y H. Jiménez (Comp.), Tutoría universitaria, 13-26. La Laguna: Servicio de publicaciones de la Universidad de La Laguna.

ÁLVAREZ, M. B; LORCA, P. Y GARCÍA, J. (2010). El Programa de Acción Tutorial como complemento de la acción docente en el Espacio Europeo de Educación Superior (EEES). Revista de Educación en Contabilidad, Finanzas y Administración de Empresas, I (1), 5-19.

ÁLVAREZ, P. Y GONZÁLEZ, M. (2008). Análisis y valoración conceptual sobre las modalidades de tutoría universitaria en el espacio europeo de educación superior. Revista Interuniversitaria de Formación del Profesorado, 22(1), 49-70. Recuperado de http://www.udc.es/cufie/ufa/patt/bibliografia/TitoriaUniversitaria/Analisis\%20y\%2 0valoracion\%20conceptual\%20sobre\%20las\%20modalidades\%20de\%20tutoria\%2 0universitaria\%20en\%20EEES.pdf

ANGROSINO, M. (2012). Etnografía y observación participante en Investigación Cualitativa. Madrid: Morata.

ARBIZU, F., LOBATO, C. Y CASTILLO, L. (2005). Algunos modelos de abordaje de la tutoría universitaria. Revisa de Psicodidáctica, 10 (1), 7-22.

BARBOUR, R. (2013). Los grupos de discusión en investigación cualitativa. Madrid: Morata.

BISQUERRA, R. (coord.) (2004). Metodología de la investigación educativa. Madrid: La Muralla.

CALVO, A.; HAYA, I. Y RODRÍGUEZ-HOYOS, C. (2012). La universidad a la luz de las opiniones del alumnado. Un estudio dirigido a mejorar la acción tutorial. Actas del XII Congreso de Instituciones y Organizaciones Educativas. Celebrado en Granada, diciembre de 2012. Universidad de Granada. 
CANO, R. (2008). Modelo organizativo para la planificación y desarrollo de la tutoría universitaria en el marco del proceso de convergencia europea en educación Superior. Revista Interuniversitaria de Formación del Profesorado, 22 (1), 185206.

CASTAÑO, E.; BENITO, A., PORTELA, A. Y RODRÍGUEZ, R.M. (2007). Repercusiones en los alumnos de primer curso de la implantación del Espacio Europeo. Revista Complutense de Educación, 18 (1), 199-216. Recuperado de http://revistas.ucm.es/index.php/RCED/article/view/RCED0707120199A/15761

CRISOL, E. (2012). Opinión y percepción del profesorado y de los estudiantes sobre el uso de las metodologías activas en la universidad de Granada. Tesis doctoral. Universidad de Granada. Recuperado de http://0hera.ugr.es.adrastea.ugr.es/tesisugr/21224043.pdf

DE LA IGLESIA, M C. (2012). La identificación de factores en el desarrollo de competencias de los estudiantes universitarios. Un estudio exploratorio. Revista Complutense de Educación, 23 (3), 207-240. Recuperado de http://revistas.ucm.es/index.php/RCED/article/view/39110

DOMíNGUEZ, G.; ÁLVAREZ, F. J. Y LÓPEZ, A. M. (2013). Acción tutorial y orientación en el período de transición de la Educación Secundaria a la Universidad. La orientación al alumnado de nuevo ingreso. REDU. Revista de Docencia Universitaria, 11 (2), 221-241. Recuperado de http://redu.net/redu/index.php/REDU/article/view/439

DOPICO, E. (2013). Tutoría universitaria: propuestas didácticas de competencia tutorial. REDU. Revista de Docencia Universitaria, 11 (2), 195-220. Recuperado de http://red-u.net/redu/index.php/REDU/article/view/370

ESCUDERO, T. (2000). La evaluación y mejora de la enseñanza en la universidad: otra perspectiva. Revista de Investigación Educativa, 18 (2), 405-416.

FERNÁNDEZ-SIERRA, J. (1993). Orientación profesional y currículum de secundaria: la educación socio-laboral y profesional de los jóvenes. Málaga: Aljibe.

FLICK, U. (2009). Introducción a la investigación cualitativa. Madrid: Morata.

GAIRÍN, J., et al. (2004). La tutoría académica en el escenario europeo de la Educación Superior. Revista Interuniversitaria de Formación del Profesorado, 18 (1), 61-77.

GARCÍA, B. (2011). La tutoría en la universidad: percepción de alumnado y profesorado. Santiago de Compostela: Universidad de Santiago de Compostela.

GARCÍA, A. J. Y TROYANO, Y. (2009). El Espacio Europeo de Educación Superior y la figura del profesor tutor en la Universidad. Red, 3, 1-10.

GARCÍA NIETO, N. et al. (2005). La tutoría universitaria ante el proceso de armonización europea. Revista de Educación, 337, 189-210. 
GARCÍA NIETO, N. (2008). La función tutorial de la Universidad en el actual contexto de la Educación Superior. Revista Interuniversitaria de Formación del Profesorado, 22 (1). 21-48.

GINÉ, N. (2009). Cómo mejorar la docencia universitaria: El punto de vista del estudiantado. Revista Complutense de Educación, 20 (1), 117-134. Recuperado de http://revistas.ucm.es/index.php/RCED/article/view/16257

HAYES, S. Y LJUNGBERG, M. (2011). Dialogic exchanges and the negotiation of differences: female graduate students' experiences of obstacles related to academic mentoring. The qualitative report. Vol. 16 (3). pp. 682-710

HERNÁNDEZ, F. et al. (2011). Aprender desde la indagación en la universidad. Barcelona: Octaedro.

HERRERA, L. (2011). Orientación, tutoría y mentorización en Educación Superior: Una labor destinada tanto al alumnado como al profesorado universitario. Dedica. Revista de Educação e Humanidades, 1, 425-452.

KVALE, S. (2011). Las entrevistas en Investigación Cualitativa. Madrid, Morata.

LOBATO, C. Y LIVENTO, M. C. (2013). La Orientación y tutoría universitaria: una aproximación actual. REDU: Revista de Docencia Universitaria, 11 (2), 17-25. Recuperado de http://red-u.net/redu/index.php/REDU/article/view/646/417

MARTÍNEZ MUÑOZ, M. (2009). La orientación y la tutoría en la universidad en el marco del Espacio Europeo de Educación Superior (EEES). Revista Fuentes, 9, 7897.

MARTÍNEZ SERRANO, M. C. (2009). La tutoría universitaria ante la creación del Espacio Europeo de Educación Superior. XXI, Revista de Educación, 11, 235-244. Recuperado http://www.uhu.es/publicaciones/ojs/index.php/xxi/article/viewFile/548/812

PÉREZ CUSO, F.J. (2013). Tutoría universitaria: ¿Un elemento de calidad? Un estudio en la Facultad de Educación de la Universidad de Murcia. Tesis doctoral. Universidad de Murcia. Recuperado de: http://www.tdx.cat/bitstream/handle/10803/123972/TFJPC.pdf?sequence=1

RODRÍGUEZ, S. (Coord.) (2004). Manual de tutoría universitaria. Recursos para la acción. Madrid: Octaedro.

RODRÍGUEZ ROMERO, M. M. (2003). Las metamorfosis del cambio educativo. Madrid: Akal.

RUIZ DE MIGUEL, C. Y OLIVEROS, L. (2006). La opinión del alumnado de la Facultad de Educación (UCM) acerca de la metodología docente. Revista Complutense de Educación, 17 (1), 29-48. Recuperado de http://revistas.ucm.es/index.php/RCED/article/view/RCED0606120029A/15861 
RUMBO, B. Y GÓMEZ T. F. (2011). La acción tutorial en un contexto universitario masificado y la reivindicación europea de su valor formativo. Revista de Formación e Innovación Educativa Universitaria, 4 (1), 13-34.

SANDÍN, M. (2003). Investigación cualitativa en educación: fundamentos y tradiciones. Madrid: McGraw-Hill.

SEALE, J. (2010): Doing student voice works in higher education: an exploration of the value of participatory methods. British Educational Research Journal, 36(6), pp. 995-1015.

SOBRADO, L. (2008). Plan de acción tutorial en los centros docentes universitarios: el rol del profesor tutor. Revista Electrónica Interuniversitaria de Formación del Profesorado, 22 (1), 89-107.

SOLA, T. Y MORENO, A. (2005). La acción tutorial en el contexto del Espacio Europeo de Educación Superior. Educación y Educadores, 8, 123-143. Recuperado de http://educacionyeducadores.unisabana.edu.co/index.php/eye/article/view/571

VALLEJO, M. Y MOLINA, J. (2011). Análisis de las metodologías activas en el grado de maestro en educación infantil: la perspectiva del alumnado. Revista Electrónica Interuniversitaria de Formación del Profesorado, 14 (1), 207-217.

\section{Correspondencia con los autores}

Carlos RODRÍGUEZ-HOYOS

Departamento de Educación. Universidad de Cantabria

Avda. Los Castros, s/n

39005 Santander

e-mail: rodriguezhc@unican.es

\section{Adelina CALVO SALVADOR}

Departamento de Educación. Universidad de Cantabria

Avda. Los Castros, s/n

39005 Santander

e-mail: calvoa@unican.es

\section{Ignacio HAYA SALMÓN}

Departamento de Educación. Universidad de Cantabria

Avda. Los Castros, s/n

39005 Santander

e-mail: hayai@unican.es 